\title{
Performance Optimization of Distributed Primal-Dual Algorithm over Wireless Networks
}

\author{
Zhaohui Yang*, Mingzhe Chen ${ }^{\dagger \ddagger}$, Kai-Kit Wong ${ }^{\S}$, H. Vincent Poor $^{\dagger}$, and Shuguang Cui ${ }^{\ddagger}$ \\ ${ }^{*}$ Department of Engineering, King's College London, WC2R 2LS, UK. \\ $\dagger^{\dagger}$ Electrical Engineering Department, Princeton University, NJ, 08544, USA. \\ ${ }_{\ddagger}^{\ddagger}$ Shenzhen Research Institute of Big Data and School of Science and Engineering, the Chinese University of Hong Kong, \\ Shenzhen, 518172, China. \\ $\S$ Department of Electronic and Electrical Engineering, University College London, London, United Kingdom. \\ Emails: yang.zhaohui@kcl.ac.uk, mingzhec@ princeton.edu, kai-kit.wong@ucl.ac.uk, \\ robert.cui@gmail.com,poor@princeton.edu.
}

\begin{abstract}
In this paper, the problem of convergence rate optimization for distributed primal-dual algorithm over wireless communications is investigated. In the considered model, each user locally updates the primal and dual variables, which are uploaded to the base station (BS). The BS aggregates the data from the users and broadcast the aggregated value to all users. This resource allocation problem is formulated as an optimization problem whose goal is to minimize the gap between the optimal value and the obtained value after a fixed number of iterations in distributed primal-dual algorithm. To solve this problem, the convergence rate is obtained in closed form for the primal-dual algorithm with considering the impact of wireless factors. Based on this convergence rate, the optimal condition for the power control and resource block allocation is obtained. An iterative algorithm with low complexity is proposed to solve this joint power control and resource block allocation problem. Simulation results show that the proposed algorithm can achieve better compared to baseline methods.
\end{abstract}

Index Terms-Dual method, convergence rate, resource allocation.

\section{INTRODUCTION}

Recently, the security concern and the availability of abundant data and computation resources in wireless networks are pushing the deployment of optimization algorithms towards the network edge [1]. This has led to a significant interest in distributed optimization methods. In the distributed optimization, each node can compute the data and sends the results to its neighbours or the center. Distributed optimization has many applications, such as channel estimation [2], trajectory optimization, and user behaviour prediction [3].

Distributed optimization algorithms have two main classes: distributed primal algorithm [4]-[7] and distributed primaldual algorithm [8]-[12]. In [4], the authors proposed fast distributed gradient algorithms to minimize the sum of individual cost function. The decentralized gradient descent method was proposed in [7], where all agents collaborate with their neighbors through information exchange. Compared to distributed primal algorithm, it was shown that distributed primal-dual algorithm has better performance in terms of fast convergence rate [8]. The distributed alternating direction method of multipliers (ADMM) was proposed in [8] for solving separable optimization problems. For distributed optimization with global inequality constraints, the authors in [9] studied deterministic and stochastic primal-dual subgradient algorithms. To reduce the communication cost of a decentralized algorithm, [10] proposed a communicationcensored ADMM. A variant ADMM algorithm was proposed in [11], which has less communication overhead but with the same convergence rate of standard ADMM. To further reduce the communication overhead, the authors in [12] investigated coding for stochastic incremental distributed primal-dual algorithm. However, the above distributed primal-dual works [8]-[12] all ignored the affect of wireless factors (such as transmission error) when implementing distributed primal-dual algorithm over wireless communications.

The main contribution of this paper is a framework for optimizing primal-dual algorithm over wireless networks. In particular, we formulate a joint resource allocation and power control problem aiming to minimize the error of the primaldual algorithm. Considering the affect of wireless factors, the convergence rate of the primal-dual algorithm is analysed.

The rest of this paper is organized as follows. System model and problem formulation are described in Section II. Section III provides the convergence analysis and resource allocation. Simulation results are presented in Section IV. Conclusions are drawn in Section V.

\section{System Model and Problem Statement}

Considering a distributed computing network with a set $\mathcal{N}$ of $N$ users and one base station (BS). Each user $n$ has a local dataset $\mathcal{D}_{n}$. Due to data privacy issue, only user $n$ can access dataset $\mathcal{D}_{n}$.

\section{A. Primal-Dual Model}

We consider the distributed primal-dual algorithm for solving the optimization problem:

$$
\begin{array}{ll}
\min _{\boldsymbol{x}} & f(\boldsymbol{x}) \triangleq \frac{1}{N} \sum_{n=1}^{N} f_{n}\left(\boldsymbol{x}, \mathcal{D}_{n}\right) \\
\text { s.t. } & g_{m}(\boldsymbol{x}) \leq 0, \quad \forall m \in \mathcal{M},
\end{array}
$$

where $f_{n}\left(\boldsymbol{x}, \mathcal{D}_{n}\right)$ and $g_{m}(\boldsymbol{x})$ are convex functions, $N$ is the total number of users, $\mathcal{M}=\{1, \cdots, M\}$, and $M$ is 
the number of constraints. For notational simplicity, we use $f_{n}\left(\boldsymbol{x}, \mathcal{D}_{n}\right)$ to denote $f_{n}(\boldsymbol{x})$ in the following.

To provide the distributed primal-dual algorithm, the Lagrange function of problem (1) can be given by:

$$
\begin{aligned}
\mathcal{L}(\boldsymbol{x}, \boldsymbol{\lambda}) & =\frac{1}{N} \sum_{n=1}^{N} f_{n}(\boldsymbol{x})+\sum_{m=1}^{M} \lambda_{m} g_{m}(\boldsymbol{x}) \\
& =\frac{1}{N} \sum_{n=1}^{N}\left(f_{n}(\boldsymbol{x})+\sum_{m=1}^{M} \lambda_{m} g_{m}(\boldsymbol{x})\right)
\end{aligned}
$$

where $\boldsymbol{\lambda}=\left[\lambda_{1}, \cdots, \lambda_{M}\right]^{T}$ is the Lagrange multiplier associated with constraint (1a). For each user $n$, we define the local Lagrange function

$$
\mathcal{L}_{n}(\boldsymbol{x}, \boldsymbol{\lambda})=f_{n}(\boldsymbol{x})+\boldsymbol{\lambda}^{T} \boldsymbol{g}(\boldsymbol{x}),
$$

where $\boldsymbol{g}(\boldsymbol{x}) \triangleq\left[g_{1}(\boldsymbol{x}), \cdots, g_{M}(\boldsymbol{x})\right]^{T}$. The sub-gradients of local Lagrange function can be given as follows:

$$
\nabla_{\boldsymbol{x}} \mathcal{L}_{n}(\boldsymbol{x}, \boldsymbol{\lambda})=\nabla f_{n}(\boldsymbol{x})+\boldsymbol{\lambda}^{T} \nabla \boldsymbol{g}(\boldsymbol{x}),
$$

and

$$
\nabla_{\boldsymbol{\lambda}} \mathcal{L}_{n}(\boldsymbol{x}, \boldsymbol{\lambda})=\boldsymbol{g}(\boldsymbol{x}) .
$$

Based on the definition of local Lagrange function, the distributed primal-dual algorithm is proposed to solve the following minmax problem [9]:

$$
\max _{\boldsymbol{\lambda}} \min _{\boldsymbol{x}} \frac{1}{N} \sum_{n=1}^{N} \mathcal{L}_{n}(\boldsymbol{x}, \boldsymbol{\lambda})
$$

The distributed primal-dual algorithm is given in Algorithm 1. In Algorithm 1, each user updates the dual variable $\boldsymbol{\lambda}(t+1)$ and obtains a copy of the primal variable $\boldsymbol{y}_{n}(t+1)$. Note that $\alpha(t)$ is a dynamic step size for the sub-gradient descend procedure. The BS aggregates the obtained copies of primal variables from all users and broadcasts this aggregated value to all users. After a sufficient number of iterations, such as $T$ iterations, each user can obtain the estimation of the optimal primal variable as in (5).

\section{B. Wireless Communication Model}

For the uplink transmission, orthogonal frequency division multiple access (OFDMA) technique is applied and each user is assigned with one resource block (RB). Assume that there are a total number of $N$ RBs. Assume that there are $N$ RBs. Let $a_{l n} \in\{0,1\}$ denote the RB association, i.e., $a_{l n}=1$ means that RB $l$ is assigned to user $n$ and $a_{l n}=0$ otherwise. Due to the fact that each user can be assigned with only one $\mathrm{RB}$ and each RB should be occupied by only one user, we have

$$
\sum_{l=1}^{N} a_{l n}=1, \sum_{n=1}^{N} a_{l n}=1 .
$$

When user $n$ is assigned with $\mathrm{RB} l$, the uplink transmission rate of user $n$ is

$$
r_{l n}=B \log _{2}\left(1+\frac{p_{n} \beta_{l} d_{n}^{-\zeta} o_{n}}{I_{l}+B N_{0}}\right),
$$

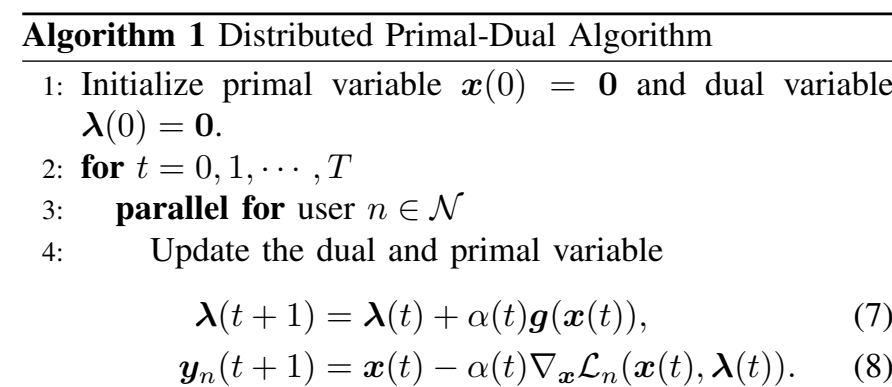

5: $\quad$ Each user sends $\boldsymbol{y}_{i}(t)$ to the BS.

end for

The BS computes

$$
\boldsymbol{x}(t+1)=\frac{1}{N} \sum_{n=1}^{N} \boldsymbol{y}_{n}(t+1)
$$

and broadcasts the value to all users.

8: $\quad$ Set $t=t+1$.

9: end for

10: Output weighted average value of the primal variable

$$
\hat{\boldsymbol{x}}(T)=\frac{\sum_{t=0}^{T-1} \alpha(t) \boldsymbol{x}(t)}{\sum_{t=0}^{T-1} \alpha(t)} .
$$

where $B$ is the bandwidth of each $\mathrm{RB}, p_{n}$ is the transmission power of user $n, \beta_{l}$ is the reference channel gain between user and the BS on $\mathrm{RB} l$ at the reference distance $1 \mathrm{~m}, d_{n}$ is the distance between user $n$ and the $\mathrm{BS}, \zeta$ is pathloss factor, $o_{n} \sim \exp (1)$ is the small scale fading.

Due to the randomness of wireless communication cannel, the user may transmit data with error. For user $n$ with $\mathrm{RB} l$, the error rate is defined as

$$
q_{l n}=\mathbb{P}\left(r_{l n}<R\right),
$$

where $R$ is the minimum rate for uploading the updated primal variable to the BS. To calculate the value of $q_{l n}$, we have the following lemma.

Lemma 1. The data error rate of user $n$ with $R B l$ is

$$
q_{l n}=1-\exp \left(-\frac{D_{l n}}{p_{n}}\right)
$$

where $D_{l n}=\frac{\left(2^{R / B}-1\right)\left(I_{l}+B N_{0}\right)}{\beta_{l} d_{n}^{-\zeta}}$.

Proof: Based on (12) and (13), we have

$$
\begin{aligned}
q_{l n} & =\mathbb{P}\left(r_{l n}<R\right) \\
& =\mathbb{P}\left(o_{n}<\frac{\left(2^{R / B}-1\right)\left(I_{l}+B N_{0}\right)}{p_{n} \beta_{l} d_{n}^{-\zeta}}\right) \\
& =1-\exp \left(-\frac{\left(2^{R / B}-1\right)\left(I_{l}+B N_{0}\right)}{p_{n} \beta_{l} d_{n}^{-\zeta}}\right),
\end{aligned}
$$

where the last equality follows from $o_{n} \sim \exp (1)$. 
Since user $n$ can occupy any one $\mathrm{RB}$, the data error rate of user $n$ is

$$
q_{n}=\sum_{l=1}^{N} a_{l n} q_{l n}
$$

In the considered system, if the received primal variable $\boldsymbol{y}_{n}$ from user $n$ contains errors, the BS will not use it for the update of the aggregated primal variable. Let $C_{n}(t) \in$ $\{0,1\}$ indicate that whether user $n$ transmits primal variable $\boldsymbol{y}_{n}$ in time $t$ contains error or not. In particular, $C_{n}(t)=1$ shows that $\boldsymbol{y}_{n}$ received by the BS does not contains any data error; otherwise, we have $C_{n}(t)=0$. The BS computes the aggregated primal variable as ${ }^{1}$

$$
\boldsymbol{x}(t+1)=\frac{\sum_{n=1}^{N} C_{n}(t) \boldsymbol{y}_{n}(t+1)}{\sum_{n=1}^{N} C_{n}(t)}
$$

where

$$
C_{n}(t)=\left\{\begin{array}{ll}
1, & \text { with probability } 1-q_{n} \\
0, & \text { with probability } q_{n}
\end{array} .\right.
$$

\section{Problem Formulation}

We aim to jointly optimize the RB allocation and power control for all users to minimize the gap of the estimation and the optimal value in distributed primal-dual algorithm, which is given as

$$
\begin{array}{ll}
\min _{\boldsymbol{A}, \boldsymbol{p}} & \mathbb{E}\left(f(\hat{\boldsymbol{x}}(T))-f\left(\boldsymbol{x}^{*}\right)\right) \\
\text { s.t. } & \sum_{l=1}^{N} a_{l n}=1, \quad \forall l \in \mathcal{N}, \\
& \sum_{n=1}^{N} a_{l n}=1, \quad \forall n \in \mathcal{N}, \\
& \sum_{n=1}^{N} p_{n} \leq P_{\max }, \\
& a_{l n} \in\{0,1\}, \quad \forall l, n \in \mathcal{N}, \\
& 0 \leq p_{n} \leq P_{n}, \quad \forall n \in \mathcal{N},
\end{array}
$$

where $\boldsymbol{A}=\left\{a_{l n}\right\}_{N \times N}, \boldsymbol{p}=\left[p_{1}, \cdots, p_{N}\right]^{T}, \mathbb{E}(f(\hat{\boldsymbol{x}}(T))-$ $\left.f\left(\boldsymbol{x}^{*}\right)\right)$ denotes the gap of the estimation and the optimal value in Algorithm 1, $P_{\max }$ is the maximum total transmission power of all users, and $P_{n}$ is the maximum transmission power of user $n$. Constraints (19a) and (19b) indicate that each user can occupy only one RB and each RB can be assigned with only one user. Constraint (19a) shows that the total transmission of all users cannot exceed a preferred value, which can guarantee that the energy consumption of the whole system is limited.

\footnotetext{
${ }^{1}$ Note that the denominator in (17) is zero only for the case that $C_{n}(t)=0$ for all $n$ with probability $\Pi_{n=1}^{N} q_{n}$. Since the probability $\Pi_{n=1}^{N} q_{n}$ approaches zero when the number of users is large, we ignore the case that $C_{n}(t)=0$ for all $n$.
}

\section{CONVErgence Analysis And Resource ALLOCATION}

\section{A. Convergence Analysis}

To analyze the convergence rate of Algorithm 1, we make the following three assumptions:

Assumption 1. Compact Feasible Set: The feasible set of primal variable $\boldsymbol{x}$ satisfying (1a) is non-empty, compact, and convex. Denote $R$ as the smallest radius of the $\ell_{2}$ ball with original center that contains the feasible set, i.e., $\|\boldsymbol{x}\| \leq R$ for all $\boldsymbol{x}$ satisfying (1a). Furthermore, this feasible set is known by all users.

Assumption 2. Slater Condition: There exists a solution $\boldsymbol{x}$ such that $g_{m}(\boldsymbol{x})<0, \forall m \in \mathcal{M}$.

Assumption 2 indicates that the primal problem in (1) and the dual problem (6) have the same optimal objective value, and the optimal dual variable $\boldsymbol{\lambda}^{*}$ has a finite value. Denote $S$ as the finite maximum value for $\lambda_{m}(t)$, i.e., $\lambda_{m}(t)<S$.

Assumption 3. Lipschitz Continuous: Both functions $f_{n}(\boldsymbol{x})$ and $g_{m}(\boldsymbol{x})$ are convex on the feasible set, and the first-order derivative of functions $f_{n}(\boldsymbol{x})$ and $g_{m}(\boldsymbol{x})$ are bounded by $L$, i.e.,

$$
\nabla f_{n}(\boldsymbol{x}) \leq L, \nabla g_{m}(\boldsymbol{x}) \leq L, \quad \forall n \in \mathcal{N}, m \in \mathcal{M}
$$

where $L<\infty$ is a constant.

Based on the above assumptions, we have the following theorem about the convergence of Algorithm 1.

Theorem 1. If we run Algorithm 1 with $T$ iterations, we have

$$
\mathbb{E}\left(f(\hat{\boldsymbol{x}}(T))-f\left(\boldsymbol{x}^{*}\right)\right) \leq \frac{R^{2}+\sum_{n=1}^{N} d_{1}\left(1-q_{n}\right)}{d_{2}\left(1-q_{0}\right)}
$$

where $d_{1}=\sum_{t=0}^{T-1}\left(L+L M S+M L^{2} R^{2}\right) \alpha(t)^{2}, d_{2}=$ $2 N \sum_{t=0}^{T-1} \alpha(t)$ and $q_{0}=\max _{n \in \mathcal{N}} q_{n}$.

Proof: Please refer to Appendix A.

Theorem 1 provides an upper bound of the gap between the estimated value and the optimal value. If we choose the step size $\alpha(t)$ (for example $\alpha(t)=1 / T$ ) satisfying $\sum_{t=0}^{\infty} \alpha(t)=$ $\infty$ and $\sum_{t=0}^{\infty} \alpha(t)^{2}<\infty$, we have $\lim _{T \rightarrow \infty} \mathbb{E}(f(\hat{\boldsymbol{x}}(T))-$ $\left.f\left(\boldsymbol{x}^{*}\right)\right)=0$, which shows that $\hat{\boldsymbol{x}}(T)$ approaches the optimal solution.

\section{B. Resource Allocation}

Based on Theorem 1, problem (19) can be reformulated as

$$
\begin{array}{ll}
\min _{\boldsymbol{A}, \boldsymbol{p}} & \frac{R^{2}+\sum_{n=1}^{N} d_{1}\left(1-q_{n}\right)}{d_{2}\left(1-\max _{n \in \mathcal{N}} q_{n}\right)} \\
\text { s.t. } & (19 a)-(19 e)
\end{array}
$$

To solve problem (22), we have the following lemma about the optimal condition. 
Lemma 2. For the optimal solution $\left(\boldsymbol{A}^{*}, \boldsymbol{p}^{*}\right)$ of problem (22), we always have

$$
q_{1}^{*}=\cdots=q_{N}^{*} .
$$

Proof: Assume that the optimal solution of problem (22) is $\left(\boldsymbol{A}^{*}, \boldsymbol{p}^{*}\right)$ and there exists $i$ and $j$ such that $q_{i}^{*}<q_{j}^{*}$. We construct a new solution $\left(\boldsymbol{A}^{*}, \overline{\boldsymbol{p}}\right)$ with

$$
\bar{p}_{n}=p_{n}^{*}, \bar{p}_{i}=p_{i}^{*}-\epsilon \quad \forall n \neq i,
$$

where $\epsilon>0$ is a small positive constant with satisfying $q_{i}^{*}<$ $\bar{q}_{i}<q_{j}^{*}$. Since the new solution $\left(\boldsymbol{A}^{*}, \overline{\boldsymbol{p}}\right)$ is feasible and has lower objective value compared to solution $\left(\boldsymbol{A}^{*}, \boldsymbol{p}^{*}\right)$, which contradicts that solution $\left(\boldsymbol{A}^{*}, \boldsymbol{p}^{*}\right)$ is optimal. As a result, the optimal condition (23) always holds for problem (22).

Based on the optimal condition (23), the objective function in (22) is equivalent to $\frac{R^{2}+\sum_{n=1}^{N} d_{1}\left(1-q_{n}\right)}{d_{2}\left(1-\max _{n \in \mathcal{N}} q_{n}\right)}=$ $\frac{R^{2}}{d_{2}\left(1-\max _{n \in \mathcal{N}} q_{n}\right)}+N d_{1}$. Besides minimize $\frac{\left.R^{2} q_{n}\right)}{d_{2}\left(1-\max _{n \in \mathcal{N}} q_{n}\right)}$ is equal to minimize $\max _{n \in \mathcal{N}} q_{n}$ ). Consequently, problem (22) can be simplified as

$$
\begin{array}{ll}
\min _{\boldsymbol{A}, \boldsymbol{p}, q} & q \\
\text { s.t. } & q \geq 1-\sum_{l=1}^{n} a_{l n} \exp \left(-\frac{D_{l n}}{p_{n}}\right), \quad \forall n \in \mathcal{N}, \\
& (19 a)-(19 e),
\end{array}
$$

where inequality (25a) holds with equality for the optimal solution as otherwise the objective value can be further improved. To solve problem (25), we use an iterative method, which optimizes $\boldsymbol{A}$ and $\boldsymbol{p}$ in an alternating manner.

Give power vector $\boldsymbol{p}$, problem (25) is a mixed linear integer problem. By temporally relaxing integer variable $a_{l n} \in[0,1]$, problem (25) with fixed $\boldsymbol{p}$ is a standard linear problem, which can be effectively solved via the simplex method. Then, we can obtain the integer value of $a_{l n}$ by using the rounding method.

With fixed RB association $\boldsymbol{A}$, problem (25) reduces to

$$
\begin{array}{ll}
\min _{\boldsymbol{p}, q} & q \\
\text { s.t. } & q \geq 1-\exp \left(-\frac{D_{l_{n} n}}{p_{n}}\right), \quad \forall n \in \mathcal{N}, \\
& \sum_{n=1}^{N} p_{n} \leq P_{\max }, \\
& 0 \leq p_{n} \leq P_{n}, \quad \forall n \in \mathcal{N},
\end{array}
$$

where $l_{n}$ is the assigned $\mathrm{RB}$ for user $n$, i.e., $a_{l_{n} n}=1$. According to Lemma 2, constraint (26a) holds with equality for the optimal solution and we can obtain

$$
p_{n}^{*}=-\frac{D_{l_{n} n}}{\ln \left(1-q^{*}\right)} .
$$

Substituting $p_{n}^{*}$ into constraints (26a)-(26b), the optimal $q^{*}$ should satisfy

$$
\sum_{n=1}^{N}-\left.\frac{D_{l_{n} n}}{\ln \left(1-q^{*}\right)}\right|_{n} ^{P} \leq P_{\max },
$$

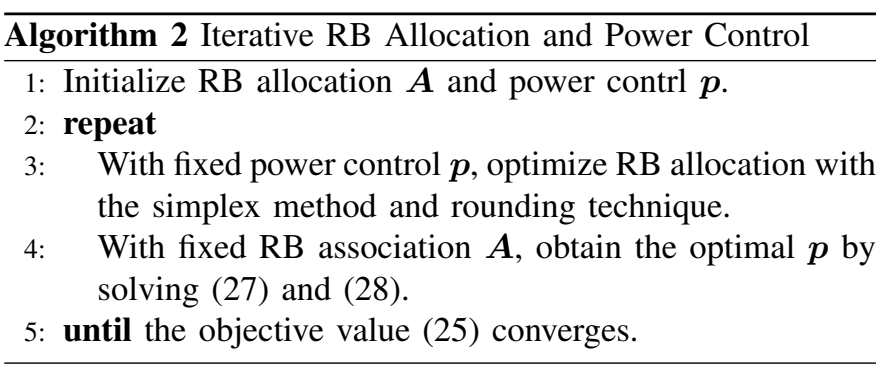

where $\left.a\right|^{b}=\min \{a, b\}$. Since the left hand-side of (28) is a decreasing function with respect to $q^{*}$, the minimal $q^{*}$ satisfying (28) can be effectively obtained by using the bisection method.

\section{Complexity Analysis}

The iterative algorithm for solving problem (22) is provided in Algorithm 2. The major complexity in each iteration lies in solving the RB allocation subproblem and the power control subproblem. With fixed power control, the complexity of using the simplex method is $\mathcal{O}\left(N^{3}\right)$ [13] for solving (25). With fixed RB association, the complexity of solving (28) with the bisection method is $\mathcal{O}(N \log (1 / \epsilon))$, where $\epsilon$ is the accuracy of the bisection method. As a result, the total complexity of Algorithm 2 is $\mathcal{O}\left(T_{0} N^{3}+T_{0} N \log (1 / \epsilon)\right)$, where $T_{0}$ is the number of iterations in Algorithm 2.

\section{Simulation Results}

In the simulations, there are $N=50$ users uniformly in a square area of size $500 \mathrm{~m} \times 500 \mathrm{~m}$ with the BS at the center. The path loss model is $128.1+37.6 \log _{10} d$ ( $d$ is in $\mathrm{km}$ ) and the standard deviation of shadow fading is $8 \mathrm{~dB}$. The bandwidth of each $\mathrm{RB}$ is $1 \mathrm{MHz}$ and the noise power spectral density is $N_{0}=-174 \mathrm{dBm} / \mathrm{Hz}$. To show the performance of the primal-dual algorithm, we consider the following classification problem [9]

$$
\begin{array}{cl}
\min _{\boldsymbol{x} \in \mathbb{R}^{d}} & f(\boldsymbol{x}) \triangleq \frac{1}{N} \sum_{n=1}^{N} \log \left(1+\exp \left(b_{n} \boldsymbol{a}_{n}^{T} \boldsymbol{x}\right)\right) \\
\text { s.t. } & g_{m}(\boldsymbol{x})=-l-x_{m}, \quad \forall m=1, \cdots, d, \\
& g_{m+d}(\boldsymbol{x})=-l-x_{m}, \quad \forall m=1, \cdots, d, \\
& \|\boldsymbol{x}\| \leq 1,
\end{array}
$$

where $\boldsymbol{a}_{n} \in \mathbb{R}^{d}$ and $b_{n} \in\{-1,1\}$.

The convergence of the distributed primal-dual algorithm is shown in Fig. 1. From this figure, we find that that the distributed primal-dual algorithm has an oscillatory behavior. When the number of users is large, the amplitude of oscillatory behavior is obvious.

We compare the proposed Algorithm 2 to solve problem (25) with two baselines: the fixed power control algorithm with only optimizing RB allocation (labelled as 'FPC') and the fixed RB allocation algorithm with only optimizing power control (labelled as 'FRBA'). Fig. 2 illustrates the maximum transmission error among all user versus the maximum sum transmit power. From this figure, the maximum transmission 


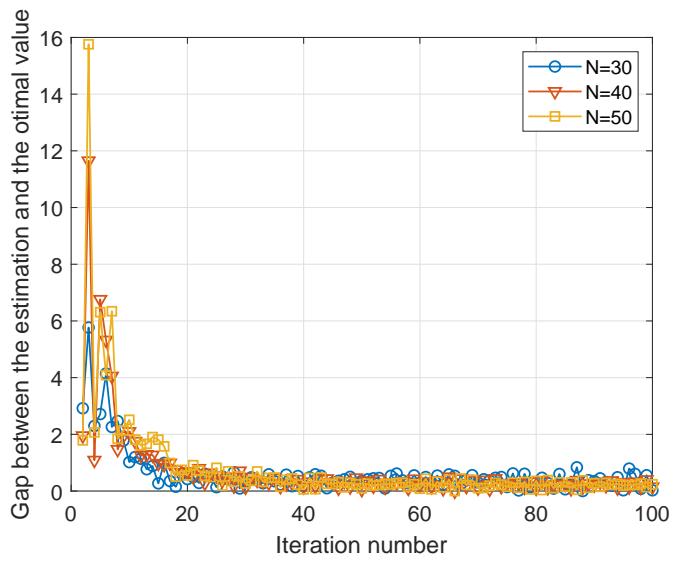

Fig. 1. Convergence behaviour of the distributed primal-dual algorithm.

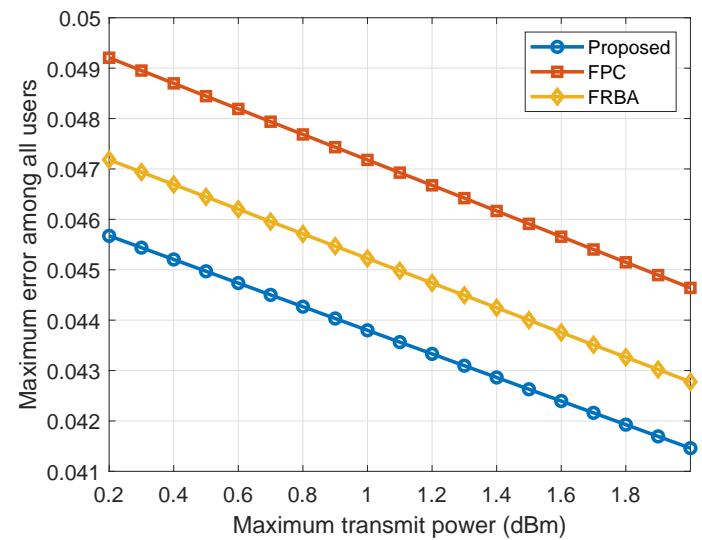

Fig. 2. Maximum transmission error among all user versus the maximum sum transmit power.

error decreases for all schemes as the maximum sum transmit power varies. This is because high transmit power can decrease the transmission error. It is observed that the proposed algorith$\mathrm{m}$ achieves the best performance, which shows the superiority of the joint power control and RA allocation design.

\section{CONCLUSIONS}

In this paper, we have investigated the convergence optimization problem of distributed primal-dual optimization over wireless communication networks via jointly optimizing RB allocation and power control. We derived the closed-form expression of the expected convergence rate of distributed primal-dual algorithm that considers the transmission error over wireless communications. Based on this convergence rate, we first obtain the optimal condition for the resource allocation. Then, an iterative algorithm is proposed, where the closed-form solution is obtained for power control subproblem. Simulation results show the superiority of the proposed solution.
APPENDIX A

ProOf OF THEOREM 1

$$
\begin{aligned}
& \mathbb{E}\left\|\boldsymbol{x}(t+1)-\boldsymbol{x}^{*}\right\|^{2} \\
\stackrel{(17)}{=} \mathbb{E}\left\|\frac{\sum_{n=1}^{N} C_{n}(t) \boldsymbol{y}_{n}(t+1)}{\sum_{n=1}^{N} C_{n}(t)}-\boldsymbol{x}^{*}\right\|^{2} & \stackrel{(8)}{=} \mathbb{E}\left\|\frac{\sum_{n=1}^{N} C_{n}(t)\left(\boldsymbol{x}(t)-\alpha(t) \nabla_{\boldsymbol{x}} \mathcal{L}_{n}(\boldsymbol{x}(t), \boldsymbol{\lambda}(t))\right)}{\sum_{n=1}^{N} C_{n}(t)}-\boldsymbol{x}^{*}\right\|^{2} \\
\stackrel{(\mathrm{a})}{\leq} & \mathbb{E} \sum_{n=1}^{N} \frac{C_{n}(t)}{\sum_{i=1}^{N} C_{i}(t)}\left\|\boldsymbol{x}(t)-\alpha(t) \nabla_{\boldsymbol{x}} \mathcal{L}_{n}(\boldsymbol{x}(t), \boldsymbol{\lambda}(t))-\boldsymbol{x}^{*}\right\|^{2} \\
= & \left\|\boldsymbol{x}(t)-\boldsymbol{x}^{*}\right\|^{2}+\mathbb{E} \sum_{n=1}^{N} \frac{C_{n}(t)}{\sum_{i=1}^{N} C_{i}(t)} \\
& \cdot\left(\alpha(t)^{2} \| \nabla_{\boldsymbol{x}} \mathcal{L}_{n}\left(\boldsymbol{x}(t), \boldsymbol{\lambda}(t) \|^{2}\right.\right. \\
& \left.-2 \alpha(t)\left(\boldsymbol{x}(t)-\boldsymbol{x}^{*}\right)^{T} \nabla_{\boldsymbol{x}} \mathcal{L}_{n}(\boldsymbol{x}(t), \boldsymbol{\lambda}(t))\right) \\
= & \left\|\boldsymbol{x}(t)-\boldsymbol{x}^{*}\right\|^{2}+\sum_{n=1}^{N} \mathbb{E}\left(\frac{C_{n}(t)}{\sum_{i=1}^{N} C_{i}(t)}\right) \\
& \cdot\left(\alpha(t)^{2} \| \nabla_{\boldsymbol{x}} \mathcal{L}_{n}\left(\boldsymbol{x}(t), \boldsymbol{\lambda}(t) \|^{2}\right.\right. \\
& \left.-2 \alpha(t)\left(\boldsymbol{x}(t)-\boldsymbol{x}^{*}\right)^{T} \nabla_{\boldsymbol{x}} \mathcal{L}_{n}(\boldsymbol{x}(t), \boldsymbol{\lambda}(t))\right),
\end{aligned}
$$

where inequality (a) follows from the fact that squared norm is a convex function. To obtain an upper bound of $\mathbb{E}\left(\frac{C_{n}(t)}{\sum_{i=1}^{N} C_{i}(t)}\right)$, we define $\kappa_{n}=\frac{C_{n}(t)}{\sum_{i=1}^{N} C_{i}(t)}$. Based on (18), we have

$$
\kappa_{n}= \begin{cases}\frac{1}{1+\sum_{i=1, i \neq n}^{N} C_{i}(t)}, & \text { with probability } 1-q_{n} \\ 0, & \text { with probability } q_{n}\end{cases}
$$

Since $\frac{1}{N} \leq \frac{1}{1+\sum_{i=1, i \neq n}^{N} C_{i}(t)} \leq 1$, we can obtain

$$
\mathbb{E}\left(\frac{C_{n}(t)}{\sum_{i=1}^{N} C_{i}(t)}\right)=\mathbb{E}\left(\kappa_{n}\right) \leq 1-q_{n} .
$$

Combining (A.1) and (A.3) yields

$$
\begin{aligned}
& \mathbb{E}\left\|\boldsymbol{x}(t+1)-\boldsymbol{x}^{*}\right\|^{2} \\
\leq & \left\|\boldsymbol{x}(t)-\boldsymbol{x}^{*}\right\|^{2}+\sum_{n=1}^{N}\left(1-q_{n}\right)\left(\alpha(t)^{2} \| \nabla_{\boldsymbol{x}} \mathcal{L}_{n}\left(\boldsymbol{x}(t), \boldsymbol{\lambda}(t) \|^{2}\right.\right. \\
& \left.-2 \alpha(t)\left(\boldsymbol{x}(t)-\boldsymbol{x}^{*}\right)^{T} \nabla_{\boldsymbol{x}} \mathcal{L}_{n}(\boldsymbol{x}(t), \boldsymbol{\lambda}(t))\right) .
\end{aligned}
$$

According to the recursion in (A.4) with $\boldsymbol{x}(0)=\mathbf{0}$, we can derive

$$
\begin{aligned}
& \mathbb{E}\left\|\boldsymbol{x}(T)-\boldsymbol{x}^{*}\right\|^{2} \\
\leq & \left\|\boldsymbol{x}^{*}\right\|^{2}+\sum_{t=0}^{T-1} \sum_{n=1}^{N}\left(1-q_{n}\right)\left(\alpha(t)^{2} \| \nabla_{\boldsymbol{x}} \mathcal{L}_{n}\left(\boldsymbol{x}(t), \boldsymbol{\lambda}(t) \|^{2}\right.\right. \\
& \left.-2 \alpha(t)\left(\boldsymbol{x}(t)-\boldsymbol{x}^{*}\right)^{T} \nabla_{\boldsymbol{x}} \mathcal{L}_{n}(\boldsymbol{x}(t), \boldsymbol{\lambda}(t))\right) .
\end{aligned}
$$


Due to the non-negativity of left-hand side in (A.5), we have

$$
\begin{gathered}
\left\|\boldsymbol{x}^{*}\right\|^{2}+\sum_{t=0}^{T-1} \sum_{n=1}^{N}\left(1-q_{n}\right) \alpha(t)^{2} \| \nabla_{\boldsymbol{x}} \mathcal{L}_{n}\left(\boldsymbol{x}(t), \boldsymbol{\lambda}(t) \|^{2}\right. \\
\geq 2 \sum_{t=0}^{T-1} \sum_{n=1}^{N}\left(1-q_{n}\right) \alpha(t)\left(\boldsymbol{x}(t)-\boldsymbol{x}^{*}\right)^{T} \nabla_{\boldsymbol{x}} \mathcal{L}_{n}(\boldsymbol{x}(t), \boldsymbol{\lambda}(t)) .
\end{gathered}
$$

According to Assumption 3, $\mathcal{L}_{n}(\boldsymbol{x}, \boldsymbol{\lambda})$ is convex with respect to $\boldsymbol{x}$ and we have

$$
\begin{aligned}
& \left(\boldsymbol{x}(t)-\boldsymbol{x}^{*}\right)^{T} \nabla_{\boldsymbol{x}} \mathcal{L}_{n}(\boldsymbol{x}(t), \boldsymbol{\lambda}(t)) \\
\geq & \mathcal{L}_{n}(\boldsymbol{x}(t), \boldsymbol{\lambda}(t))-\mathcal{L}_{n}\left(\boldsymbol{x}^{*}, \boldsymbol{\lambda}(t)\right) .
\end{aligned}
$$

Based on (A.6) and (A.7), we have

$$
\begin{aligned}
& \left\|\boldsymbol{x}^{*}\right\|^{2}+\sum_{t=0}^{T-1} \sum_{n=1}^{N}\left(1-q_{n}\right) \alpha(t)^{2} \| \nabla_{\boldsymbol{x}} \mathcal{L}_{n}\left(\boldsymbol{x}(t), \boldsymbol{\lambda}(t) \|^{2}\right. \\
& \geq 2 \sum_{t=0}^{T-1} \sum_{n=1}^{N}\left(1-q_{n}\right) \alpha(t)\left(\mathcal{L}_{n}(\boldsymbol{x}(t), \boldsymbol{\lambda}(t))-\mathcal{L}_{n}\left(\boldsymbol{x}^{*}, \boldsymbol{\lambda}(t)\right)\right) \\
& \stackrel{(3)}{=} \sum_{t=0}^{T-1} \sum_{n=1}^{N}\left(1-q_{n}\right) \alpha(t)\left(f_{n}(\boldsymbol{x}(t))-f_{n}\left(\boldsymbol{x}^{*}\right)\right) \\
& \quad+2 \sum_{t=0}^{T-1} \sum_{n=1}^{N}\left(1-q_{n}\right) \alpha(t)\left(\boldsymbol{\lambda}(t)^{T} \boldsymbol{g}(\boldsymbol{x}(t))-\boldsymbol{\lambda}(t)^{T} \boldsymbol{g}\left(\boldsymbol{x}^{*}\right)\right) \\
& \text { (b) } 2 \sum_{t=0}^{T-1} \sum_{n=1}^{N}\left(1-q_{n}\right) \alpha(t)\left(f_{n}(\boldsymbol{x}(t))-f_{n}\left(\boldsymbol{x}^{*}\right)\right) \\
& \quad+2 \sum_{t=0}^{T-1} \sum_{n=1}^{N}\left(1-q_{n}\right) \alpha(t) \boldsymbol{\lambda}(t)^{T} \boldsymbol{g}(\boldsymbol{x}(t)),
\end{aligned}
$$

where (b) follows from the fact that $g_{m}\left(\boldsymbol{x}^{*}\right) \leq 0$ and $\lambda_{m}(t) \geq$ 0 . To derive a lower bound for the last term in the right hand side of (A.8), we provide the following lemma.

Lemma 3. For all $T$, the following inequality holds

$$
\begin{aligned}
& 2 \sum_{t=0}^{T-1} \sum_{n=1}^{N}\left(1-q_{n}\right) \alpha(t) \boldsymbol{\lambda}(t)^{T} \boldsymbol{g}(\boldsymbol{x}(t)) \\
\geq & -\sum_{t=0}^{T-1} \sum_{n=1}^{N}\left(1-q_{n}\right) \alpha(t)^{2}\|\boldsymbol{g}(\boldsymbol{x}(t))\|^{2}
\end{aligned}
$$
yields

$$
\begin{aligned}
& \|\boldsymbol{\lambda}(t+1)\|^{2} \stackrel{(7)}{=}\|\boldsymbol{\lambda}(t)+\alpha(t) \boldsymbol{g}(\boldsymbol{x}(t))\|^{2} \\
= & \|\boldsymbol{\lambda}(t)\|^{2}+2 \alpha(t)(\boldsymbol{\lambda}(t))^{T} \boldsymbol{g}(\boldsymbol{x}(t))+\alpha(t)^{2}\|\boldsymbol{g}(\boldsymbol{x}(t))\|^{2} .
\end{aligned}
$$

By using the recursion method and $\boldsymbol{\lambda}(0)=\mathbf{0}$, we can obtain $\|\boldsymbol{\lambda}(T)\|^{2}=2 \sum_{t=0}^{T-1} \alpha(t)(\boldsymbol{\lambda}(t))^{T} \boldsymbol{g}(\boldsymbol{x}(t))+\sum_{t=0}^{T-1} \alpha(t)^{2}\|\boldsymbol{g}(\boldsymbol{x}(t))\|^{2}$.

Since $\|\boldsymbol{\lambda}(T)\|^{2} \geq 0$, we have

$$
\sum_{t=0}^{T-1} \alpha(t)^{2}\|\boldsymbol{g}(\boldsymbol{x}(t))\|^{2} \geq-2 \sum_{t=0}^{T-1} \alpha(t)(\boldsymbol{\lambda}(t))^{T} \boldsymbol{g}(\boldsymbol{x}(t)) .
$$

This completes the proof.

According to (A.8) and (A.9), we can obtain

$$
\begin{aligned}
& \left\|\boldsymbol{x}^{*}\right\|^{2}+\sum_{t=0}^{T-1} \sum_{n=1}^{N}\left(1-q_{n}\right) \alpha(t)^{2} \| \nabla_{\boldsymbol{x}} \mathcal{L}_{n}\left(\boldsymbol{x}(t), \boldsymbol{\lambda}(t) \|^{2}\right. \\
\geq & 2 \sum_{t=0}^{T-1} \sum_{n=1}^{N}\left(1-q_{n}\right) \alpha(t)\left(f_{n}(\boldsymbol{x}(t))-f_{n}\left(\boldsymbol{x}^{*}\right)\right) \\
& -\sum_{t=0}^{T-1} \sum_{n=1}^{N}\left(1-q_{n}\right) \alpha(t)^{2}\|\boldsymbol{g}(\boldsymbol{x}(t))\|^{2} \\
\stackrel{(\mathrm{c})}{\geq} & 2 N \sum_{t=0}^{T-1}\left(1-q_{0}\right) \alpha(t)\left(f(\boldsymbol{x}(t))-f\left(\boldsymbol{x}^{*}\right)\right) \\
& -\sum_{t=0}^{T-1} \sum_{n=1}^{N}\left(1-q_{n}\right) \alpha(t)^{2}\|\boldsymbol{g}(\boldsymbol{x}(t))\|^{2},
\end{aligned}
$$

where (c) follows from the definition $q_{0}=\max _{n \in \mathcal{N}} q_{n}$ and $f(\boldsymbol{x})=\frac{1}{N} \sum_{n=1}^{N} f_{n}(\boldsymbol{x})$.

Recall the definition of $\hat{\boldsymbol{x}}(T)$ in (10), we have

$$
\begin{aligned}
& \mathbb{E}\left(f(\hat{\boldsymbol{x}}(T))-f\left(\boldsymbol{x}^{*}\right)\right) \\
\stackrel{(\mathrm{d})}{\leq} & \frac{\sum_{t=0}^{T-1} \alpha(t)\left(f(\boldsymbol{x}(t))-f\left(\boldsymbol{x}^{*}\right)\right)}{\sum_{t=0}^{T-1} \alpha(t)} \\
\stackrel{\text { (A.10) }}{\leq} & \frac{1}{2 N\left(1-q_{0}\right) \sum_{t=0}^{T-1} \alpha(t)}\left(\left\|\boldsymbol{x}^{*}\right\|^{2}\right. \\
& +\sum_{t=0}^{T-1} \sum_{n=1}^{N}\left(1-q_{n}\right) \alpha(t)^{2} \| \nabla_{\boldsymbol{x}} \mathcal{L}_{n}\left(\boldsymbol{x}(t), \boldsymbol{\lambda}(t) \|^{2}\right. \\
& \left.+\sum_{t=0}^{T-1} \sum_{n=1}^{N}\left(1-q_{n}\right) \alpha(t)^{2}\|\boldsymbol{g}(\boldsymbol{x}(t))\|^{2}\right) \\
\stackrel{(\mathrm{e})}{\leq} & \frac{1}{2 N\left(1-q_{0}\right) \sum_{t=0}^{T-1} \alpha(t)}\left(\left\|\boldsymbol{x}^{*}\right\|^{2}\right. \\
& +\sum_{t=0}^{T-1} \sum_{n=1}^{N}\left(1-q_{n}\right) \alpha(t)^{2} \| \nabla_{\boldsymbol{x}} \mathcal{L}_{n}\left(\boldsymbol{x}(t), \boldsymbol{\lambda}(t) \|^{2}\right. \\
& \left.+\sum_{t=0}^{T-1} \sum_{n=1}^{N}\left(1-q_{n}\right) \alpha(t)^{2} M L^{2} R^{2}\right),
\end{aligned}
$$

where (d) follows from the convexity of function $f(\boldsymbol{x})$ and (e) follows from Assumption 3. To derive an upper bound for $\| \nabla_{\boldsymbol{x}} \mathcal{L}_{n}(\boldsymbol{x}(t), \boldsymbol{\lambda}(t) \|$, we provide the following lemma.

Lemma 4. The sub-gradient of the Lagrangian function is bounded by

$$
\| \nabla_{\boldsymbol{x}} \mathcal{L}_{n}\left(\boldsymbol{x}(t), \boldsymbol{\lambda}(t) \|^{2} \leq L(1+M S) .\right.
$$

Proof: From the definition of the Lagrangian function, we have

$$
\begin{aligned}
& \| \nabla_{\boldsymbol{x}} \mathcal{L}_{n}\left(\boldsymbol{x}(t), \boldsymbol{\lambda}(t)\|\stackrel{(3)}{=}\| \nabla f_{n}(\boldsymbol{x}(t))+\boldsymbol{\lambda}(t)^{T} \nabla \boldsymbol{g}(\boldsymbol{x}(t)) \|\right. \\
& \leq\left\|\nabla f_{n}(\boldsymbol{x}(t))\right\|+\sum_{m=1}^{M} \lambda_{m}(t)\left\|\nabla g_{m}(\boldsymbol{x}(t))\right\| \\
& \stackrel{\text { (f) }}{\leq} L\left(1+\|\boldsymbol{\lambda}(t)\|_{1}\right) \stackrel{\text { (g) }}{\leq} L(1+M S),
\end{aligned}
$$


where (e) follows from the triangle inequality, (f) follows from Assumption 3, (g) follows from Assumption 2.

Combining Assumption 1, (A.11) and (A.12), we can obtain equation (21).

\section{REFERENCES}

[1] W. Saad, M. Bennis, and M. Chen, "A vision of $6 \mathrm{G}$ wireless systems: Applications, trends, technologies, and open research problems," IEEE Network, 2020 (To appear).

[2] M. Kim, N.-I. Kim, W. Lee, and D.-H. Cho, "Deep learning-aided SCMA," IEEE Commun. Lett., vol. 22, no. 4, pp. 720-723, 2018.

[3] M. Chen, Z. Yang, W. Saad, C. Yin, H. V. Poor, and S. Cui, "A joint learning and communications framework for federated learning over wireless networks," arXiv preprint arXiv:1909.07972, 2019.

[4] D. Jakovetić, J. Xavier, and J. M. Moura, "Fast distributed gradient methods," IEEE Trans. Automatic Control, vol. 59, no. 5, pp. 11311146, 2014.

[5] A. Nedić and A. Olshevsky, "Distributed optimization over time-varying directed graphs," IEEE Trans. Automatic Control, vol. 60, no. 3, pp. 601-615, 2014.

[6] W. Shi, Q. Ling, G. Wu, and W. Yin, "A proximal gradient algorithm for decentralized composite optimization," IEEE Trans. Signal Process., vol. 63, no. 22, pp. 6013-6023, 2015.

[7] K. Yuan, Q. Ling, and W. Yin, "On the convergence of decentralized gradient descent," SIAM Journal on Optimization, vol. 26, no. 3, pp. 1835-1854, 2016.

[8] J. F. Mota, J. M. Xavier, P. M. Aguiar, and M. Püschel, "D-ADMM: A communication-efficient distributed algorithm for separable optimization," IEEE Trans. Signal Process., vol. 61, no. 10, pp. 2718-2723, 2013.

[9] M. B. Khuzani and N. Li, "Distributed regularized primal-dual method: Convergence analysis and trade-offs," arXiv preprint arXiv:1609.08262, 2016.

[10] Y. Liu, W. Xu, G. Wu, Z. Tian, and Q. Ling, "Communication-censored ADMM for decentralized consensus optimization," IEEE Trans. Signal Process., vol. 67, no. 10, pp. 2565-2579, 2019.

[11] A. Elgabli, J. Park, A. S. Bedi, M. Bennis, and V. Aggarwal, "Communication efficient framework for decentralized machine learning," in 2020 54 th Annual Conference on Information Sciences and Systems (CISS). IEEE, 2020, pp. 1-5.

[12] H. Chen, Y. Ye, M. Xiao, M. Skoglund, and H. V. Poor, "Coded stochastic ADMM for decentralized consensus optimization with edge computing," arXiv preprint arXiv:2010.00914, 2020.

[13] S. Boyd and L. Vandenberghe, Convex Optimization. Cambridge University Press, 2004. 